\title{
Research of the Active Components and Potential Mechanisms of Qingfei Gujin Decoction in the Treatment of Osteosarcoma Based on Network Pharmacology and Molecular Docking
}

\author{
Qingying Yan \\ Hangzhou Third People's Hospital \\ Jiewen Yang \\ Hangzhou Third People's Hospital \\ Yongwei Yao \\ Hangzhou Third People's Hospital \\ Zhen Jia \\ Hangzhou Third People's Hospital

\section{Yiqing Wang} \\ Hangzhou Third People's Hospital \\ Chen Miao \\ Hangzhou Third People's Hospital \\ Xiaobo Yan \\ Yefeng Xu ( $\nabla$ xuyefeng1008@126.com ) \\ Hangzhou Third People's Hospital
}

\section{Research Article}

Keywords: Qingfei Gujin decoction, osteosarcoma, TCM

Posted Date: March 7th, 2022

DOI: https://doi.org/10.21203/rs.3.rs-1413873/v1

License: (c) (i) This work is licensed under a Creative Commons Attribution 4.0 International License.

Read Full License 


\section{Abstract}

Aim: Qingfei Gujin decoction (QGD) has been shown to be effective against osteosarcoma. This research was aimed to investigate the main active ingredients and potential mechanisms of QGD acting on osteosarcoma through network pharmacology and molecular docking techniques.

Methods: The active ingredients and targets of QGD were screened from the TCMSP database, and the predicted targets were obtained from the PharmMapper database. Meanwhile, the targets of osteosarcoma were collected using OMIM, PharmGkb and DisGeNET databases. Then, GO and KEGG enrichment analysis was performed by RStudio. PPI and drug-ingredients-targets network were constructed using Cytoscape 3.2.1 to screen the major active ingredients, key networks and targets. Finally, molecular docking of key genes and their regulatory active ingredients was performed using AutoDockTools-1.5.6 software.

Results: 38 active ingredients were collected, generating 89 cross-targets; Quercetin, luteolin, $\beta$-sitosterol, and kaempferol were the main active ingredients of QGD acting on osteosarcoma, and major signaling pathways such as PI3K-Akt signaling pathway, MAPK signaling pathway, and IL-17 signaling pathway were observed. TP53, SRC, and ESR1 were identified as key proteins that docked well with their regulated compounds.

Conclusion: QGD is effective against osteosarcoma through multi-component, multi-target and multipathway. This study was helpful for finding effective targets and compounds for osteosarcoma treatment.

\section{Full Text}

Osteosarcoma is the most common primary bone solid malignant tumor. Local pain, followed by localized swelling and limitation of joint movement, are the typical signs and symptoms of osteosarcoma [1]. Adolescence is the highest incidence rate of osteosarcoma. The majority of cases occur in children and adolescents aged 10 to 30 , with $10 \%$ occurring in those over the age of 60 [2]. Distant metastasis can be detected in approximately $15 \%$ to $20 \%$ of patients at the initial diagnosis, with the lung being the most common metastatic site, accounting for about $85 \%$ of metastatic diseases [3]. The 5 -year survival rate for localized osteosarcoma is $67 \%$, compared with only $20 \%$ in metastatic patients, which is the leading cause of death in patients with osteosarcoma [4]. Although new targeted drugs and immune drugs have been widely used in clinics, the survival rate of osteosarcoma has not been significantly improved. Therefore, it is of great value to better understand the metastasis mechanism of osteosarcoma and find appropriate targets and drugs to prolong the survival time of osteosarcoma.

Numerous studies have shown that Traditional Chinese Medicine (TCM) can not only alleviate the symptoms of tumor patients, such as fatigue, chronic pain and cachexia, but also improve their quality of life and reduce the adverse reactions and complications caused by chemotherapy, radiotherapy and targeted therapy [5]. In China, TCM treatment runs through the treatment of tumors. 
In TCM, osteosarcoma is classified as "osteoma", " osteomyelitis" and " indurated knee mass". It is believed that osteosarcoma is frequently caused by insufficient endowment, a lack of genuine qi. Pathogenic qi enters the body, traveling with meridian qi and blood to the bone marrow, causing qi and blood stagnation, meridian obstruction, tendon erosion, and bone formation. On this basis, Qingfei Gujin Decoction (QGD, composed of Astragalus membranaceus, Fritillaria thunbergii, Platycodon grandiflorum, Hedyotis diffusa and Coicis semen) was clinically tested, and found to be have an excellent curative.

Network pharmacology, a new concept in TCM research, has been widely used to study complex network relationships between TCM and diseases. To better understand the potential mechanism of QGD in the treatment of osteosarcoma, we used network pharmacology and molecular docking technology to screen the active components of QGD and predict its possible targets and pathways in osteosarcoma, with the goal of providing a theoretical foundation for the treatment of osteosarcoma with TCM (Figure 1).

\section{Materials And Methods}

\section{The screening of active ingredients of QGD}

Traditional Chinese Medicine Systems Pharmacology Database and Analysis Platform (https://old.tcmsp-e.com/tcmsp.php ) is a one-of-a-kind pharmacological platform of Chinese herbal medicines that captures the relationships between drugs, targets and diseases [6]. We collected the potential active ingredients using the following criteria: Oral bioavailability $(O B) \geq 30 \%$, Drug like $(D L)$ $\geq 0.18$, Half-life $(\mathrm{HL}) \geq 4 \mathrm{H}$.

\section{Targets collection for QGD}

TCMSP was used to collect targets that interact with active ingredients. Simultaneously, the predicted targets of QGD were obtained using PharmMapper (http://www.lilab-ecust.cn/pharmmapper/), an online tool for identifying potential target candidates based on molecular structure[7]. All the molecular structures (mol2 format) of active ingredients were achieved from TCMSP.

\section{Targets collection for Osteosarcoma}

Candidate targets of Osteosarcoma were gathered from three major databases, OMIM (https://omim.org/), PharmGkb [8] (https://www.pharmgkb.org), and DisGeNET [9] (https://www.disgenet.org). We used the keywords "Osteosarcoma" and "Metastatic osteosarcoma" to search these databases. We chose genes with a Score $\mathbb{0} 0.04$ from the DisGeNET. To remove repeated genes and normalize the gene information, RStudio and The Practical Extraction and Report Language هPerl\were used.

\section{Drug-ingredients-targets network construction and PPI}

"Venn" package was used to analyze the intersection targets between QGD component targets and disease targets, which were considered to be the potential targets of QGD in the treatment of 
osteosarcoma. The drug-ingredients-targets network was constructed by Cytoscape software (Version 3.2.1). In this network, the active ingredients were represented by circular nodes of various colors, while potential targets were represented by rectangular nodes

The PPI network was constructed by importing the potential genes into the Search tool to retrieve interacting genes (STRING, https://string-db.org) and the organism type was selected as Homo sapiens(humans). The minimum required interaction score was set with a medium confidence $=0.9$, and all other parameters were left at their default values. The "string interactions. tsv" file was downloaded in order to visualize the network and determine the intersection of the PPI network and core genes.

The obtained "string interactions. tsv" file was imported into Cytoscape software, and any duplicated edges were removed. Betweenness centrality (BC), closeness centrality (CC), degree centrality (DC), eigenvector centrality (EC), local average connectivity-based method (LAC) were calculated using the "CytoNCA". Firstly, the top 50\% candidate genes of all values are to be as sub-networks. Secondly, the candidate genes with the top $50 \%$ of values in sub-networks are selected as critical genes and established core networks.

\section{Enrichment analysis}

R packages including "colorspace," "stringi," and "ggplot2" were installed in Rstudio, and a Bioconductor package that includes "DOSE," "clusterProfiler," and "enrichplot" was used for GO and Kyoto Encyclopedia of Genes and Genomes (KEGG) enrichment analysis.

\section{Molecular docking}

AutoDockTools-1.5.6 was used to dock the key targets and their conditioning ingredients. The mol2 chemical structure of ingredients was downloaded from the TCMSP database, and all compounds were saved as ligand parameter files in pdbqt format. In this docking process, the 3D structure of key targets was retrieved from RCSB Protein Data Bank (PDB) (www.wwpdb.org), and solvents and water molecules were removed from target protein receptor molecules using pymol software. Binding energy was used as a docking score to evaluate the protein-ligand binding potential of molecular docking. Results with value $\leq-5$ were selected and considered to have moderate binding potential and tight combination.

\section{Results}

\section{The main active ingredients and potential targets of QGD.}

38 active ingredients were selected from TCMSP database. Mol ID, molecule names, OB, DL, HL were displayed in Table 1. After summarizing the active component targets of TCMPS and the predicted targets of PharmMapper, 526 potential targets were obtained by running Perl, eliminating duplicate values and converting symbols.

\section{The Common Potential Targets Of Qgd And Osteosarcoma}


A total of 526 potential targets of osteosarcoma were collected, including 3 from OMIM, 97 from PharmGKB and 439 from DisGeNET (Fig. 2A). Finally, a total of 89 intersecting genes were screened as candidate targets to further research (Fig. 2B).

\section{Drug-ingredients-targets network and PPI}

The drug-ingredients-targets network was visualized using Cytoscape software. Figure 1C shows Hedyotis diffusa in red, Fritillaria thunbergia in rose red, Coicis semen in green, Astragalus membranaceus in light blue, Platycodon grandiflorum in dark blue. According to supplement Table 1, quercetin was associated with 77 potential targets, luteolin with 56 potential targets, $\beta$-sitosterol and kaempferol with 51 potential targets, which may be the main active ingredient of QGD for the treatment of osteosarcoma. In Fig. 1C, the components were represented by the circle in Fig. 1C, and the candidate targets were represented by the square. The greater the size of the shape, the more the components associated with it. KDR, SRC, MAPK14, HPGDS, GSK3B, MET, MMP3, HSP90AA1, GSTP1, FGFR1, ESR1, EGFR, DHFR, CHEK1, CASP3 are found to be the most frequently associated with active ingredients.

89 candidate genes were introduced into String. According to the screening conditions, the "stringinteractions. tsv" were imported into Cytoscape to establish the PPI network. The network was consisted of 82 nodes and 400 edges. The genes with BC, CC, EC, DC and LAC greater than the median were screened to construct the sub-network, which included 28 nodes and 175 edges. According to the final values of BC, CC, EC, DC and LAC (Table 2), 11 targets were obtained to become the core network, and TP53, SRC and ESR1 were considered as the key genes(Fig. 3).

\section{Go Enrichment Analysis}

Go enrichment includes biological process (BP), cellular component (CC) and molecular function (MF). 89 potential targets of QGD in the treatment of osteosarcoma were analyzed by R-package. As shown in the Fig. 4A, BP mainly included: response to steroid hormones (go: 0048545), response to metal ions (go: 0010038), cell response to oxidative stress (go: 0034599), response to radiation (go: 0009314), response to peptides (go: 1901652), response to oxidative stress (go: 0006979), regulation of apoptosis signal pathway (go: 2001233), Response to toxic substances (go: 0009636), cell response to abiotic stimuli (go: 0071214), cell response to environmental stimuli (go: 0104004); In terms of CC, it mainly included chromatin (go: 0000785), transcription factor complex (go: 0005667), membrane raft (go: 0045121), membrane micro region (go: 0098857), membrane region (go: 0098589), RNA polymerase II transcription factor complex (go: 0090575), nuclear transcription factor complex (go: 0044798), cyclin dependent protein kinase holoenzyme complex (go: 0000307), Serine /threonine protein kinase complex(go: 1902554), protein kinase complex (go: 1902911); MF mainly included: ubiquitin like protein ligase binding (go: 0044389), proximal promoter sequence specific DNA binding (go: 0000987), protein heterodimerization activity (go: 0046982), ubiquitin protein ligase binding (go: 0031625), DNA binding transcription activator activity, RNA polymerase II specificity (go: 0001228), protein tyrosine kinase activity (go: 0004713), nuclear receptor activity (go: 0004879) Transcription factor activity, direct ligand regulated sequence specific DNA binding (go: 0098531), steroid hormone receptor activity (go: 0003707), 
transmembrane receptor protein tyrosine kinase activity (go: 0004714). Based on this, QGD in the treatment of osteosarcoma might be the result of multiple mechanisms.

\section{Kegg Pathway Enrichment Analysis}

In order to further explore the possible mechanism of QGD in the treatment of osteosarcoma, we performed KEGG pathway enrichment analysis on 89 target targets. As shown in Fig. 4B. The main related pathways included PI3K Akt signal pathway, proteoglycan in cancer, MAPK signal pathway, chemical carcinogenesis receptor activation pathway, cell aging, IL-17 signal pathway, EGFR tyrosine kinase inhibitor resistance, etc.

\section{Molecular Docking Results}

Essential genes were selected for molecular docking with compounds that might regulate these targets (Supplement Table 2). The results showed that the docking binding energy of the key targets and the active ingredients were basically lower than $-5 \mathrm{Kcal} / \mathrm{mol}$. Taking the lowest binding energy for example, the results could be seen in Table 3 and the structural diagrams were shown in Fig. 5 .

\section{Discussion}

It is a global problem to prevent postoperative recurrence and distant metastasis of osteosarcoma. TCM has the benefits of safety and low toxicity, and it is increasingly being used for the prevention and treatment of osteosarcoma patients in China after surgery and chemotherapy. Network pharmacology has evolved into a sophisticated method for studying the practical components and complex mechanisms of TCM and compound prescriptions. In this study, the active ingredients of QGD were collected and network pharmacology was used to validate the effect of multi-target and multi-channel treatment.

QGD, composed of Astragalus membranaceus, Fritillaria thunbergii, Platycodon grandiflorum, Hedyotis diffusa and Coicis semen, has been shown to effectively prevent local recurrence and metastasis after osteosarcoma surgery. According to our study, the main active ingredients of QGD acting on osteosarcoma may be quercetin, luteolin, $\beta$-sitosterol, and kaempferol. Quercetin was a flavonol compound with a variety of biological activities which had been widely used to treat cardiovascular diseases, diabetes, tumors and other diseases. Maleki Dana P et al. hypothesized that quercetin inhibited osteosarcoma cell proliferation, migration and invasion, inducing autophagy and apoptosis, and could play a separate or synergistic role in overcoming drug resistance in osteosarcoma cell lines [10]. Luteolin was a kind of natural flavonoid found in many plants, and had anti-inflammatory, anti-allergic, antiviral, antitumor properties. In osteosarcoma, luteolin was involved in inhibiting tumor cell proliferation, inducing tumor cell apoptosis, affecting tumor cell cycle distribution and inhibiting tumor angiogenesis, and could be used as a tumor apoptosis sensitizer or antioxidant [11]. Meanwhile, it had been reported that luteolin could be acted as an enhancer to sensitize doxorubicin-mediated autophagy signaling in osteosarcoma cells [12]. $\beta$-sitosterol was one of the most common phytosterols. A meta-analysis 
revealed that consuming high levels of dietary $\beta$-sitosterol might have a positive effect on antitumor mechanisms [13]. Although the mechanism of $\beta$-sitosterol in osteosarcoma was rarely reported, it has been shown to improve bone fragility and fracture occurrence [14]. Kaempferol had the effects of antioxidant, anti-inflammatory, pro-apoptotic, cardioprotective and anticancer activities. In 2010, it was reported that kaempferol reduced the cell viabilities of osteosarcoma cells in a dose-dependent manner and induced apoptosis in human osteosarcoma cells via endoplasmic reticulum stress mitochondrial signaling pathways [15]. Chen $\mathrm{HJ}$ et al. further confirmed that kaempferol attenuated the MAPK signaling pathways including ERK, JNK and p38, resulting in decreased DNA binding ability of AP-1, and hence, the downregulation of the expression and enzymatic activities of MMP-2, MMP-9, and Upa, contributing to the inhibition of metastasis of osteosarcoma cells [16].

P53 was encoded by the TP53 gene on human chromosome 17 and served as a tumor suppressor gene in the human body, protecting genomic integrity. Mutation in the TP53 gene was detectable in about 50\% of patients with tumor, and more than $75 \%$ of TP53 gene mutations resulted in a loss of wild-type p53 activities, thereby promoting tumorigenesis, progression, and metastasis [17]. TP53 mutations were found in $47-90 \%$ of patients with osteosarcoma, and TP53 Patients with mutations had poor overall survival rates [18]. Data suggested that TP53 mutations had a negative impact on 2-year overall survival [19]. Therefore, targeting TP53 may be an effective strategy in the treatment of osteosarcoma in the future. SRC family kinases were the most prominent family of non-receptor tyrosine kinases. As the oldest oncogene, SRC was one of the best-studied targets for cancer therapy, which was closely related to regulating appreciation, angiogenesis, invasion metastasis, bone metabolism [20]. Src could be activated by multiple signaling pathways to become phospho-Src ( $p-S r c)$, and researchers had found that the expression of Src and p-Src in osteosarcoma was significantly higher, and the expression level was related to the clinical stage, tumor metastasis and survival time of osteosarcoma, which could be used as an auxiliary index to judge the malignant phenotype of osteosarcoma and prompt the prognosis of osteosarcoma [21]. The Src pathway was associated with osteosarcoma metastasis. About $95 \%$ of samples examined express Src or had evidence of downstream activation of this pathway. As a potent selective SRC kinase inhibitor, the clinical trials had shown Saracatinib (AZD0530) was well tolerated but had no apparent impact on overall survival of osteosarcoma [22]. ESR1, a gene that encodes estrogen receptor a (ER), had been widely studied in breast cancer. Although there were fewer studies on ESR1 and osteosarcoma, ESR1-mediated estrogen effects associated with bone mineralization have been reported [23]. One of the mechanisms associated with the poor prognosis of osteosarcoma was abnormal ESR1 methylation [24]. Our study found that the core genes of QGD in the treatment of osteosarcoma were TP53, SRC, ESR1, and molecular docking revealed that the binding capacity of the core gene to its regulated compounds was lower than $-5 \mathrm{Kcal} / \mathrm{mol}$, suggesting that QGD could be multi-targeted for osteosarcoma.

$\mathrm{PI}$ IK/AKT was one of the most important carcinogenic pathways in human cancer, and it was frequently overactivated in osteosarcoma. It was involved in tumor occurrence and progression, including proliferation, invasion, cell cycle progression, angiogenesis and chemical resistance. Therefore, targeting the PI3K/AKT signaling pathway was proposed as a potential treatment for osteosarcoma [25]. The 
MAPK signaling pathway was involved in a variety of cellular processes, and its downstream pathways were JNK, P38, and ERK. In osteosarcoma, Xue Y et al. found that iron chelators could activate the ROSrelated MAPK signaling pathway, promoting apoptosis and reducing malignant proliferation [26]. According to Zhang $L$ et al., the Chinese herb Cardamomin might inhibit the proliferation, migration and invasion of osteosarcoma by activating the P38 and MAPK signaling pathway [27]. Interleukin 17 was a CD4 + T cell-derived cytokine that stimulated some tumor cells to secrete angiogenesis factor, and the IL17 receptor might represent a marker for the osteosarcoma metastasis [28]. By enriching potential targets through KEGG, we found PI3K/AKT, MAPK, IL-17 signaling pathways were the main pathways of QGD in the treatment of osteosarcoma, indicating that QGD could inhibit the invasion and metastasis of osteosarcoma through multiple pathways.

In summary, QGD had a variety of components and played an anti-osteosarcoma role via multiple targets and pathways. Unfortunately, the efficacy of QGD in the treatment of osteosarcoma had not been validated at the molecular level, and further research was required to supplement the conclusion.

\section{Conclusion}

A total of 38 active ingredients were extracted from the QGD. 526 targets and 526 disease targets were collected, as well as 89 common targets were generated. Quercetin, luteolin, $\beta$-sitosterol, and kaempferol were the main active ingredients. We discovered that the main pathways of QGD against osteosarcoma were the PI3K-Akt signaling pathway, proteoglycans in cancer, MAPK signaling pathway, chemical carcinogenesis receptor activation pathway, cell aging and IL-17 signaling pathway, EGFR tyrosine kinase inhibitor resistance. In addition, TP53, SRC, and ESR1 were presumed to be key proteins becaused of their good docking with the regulated compounds. These findings may aid in the identification of effective targets and compounds for osteosarcoma treatment, as well as provide a particular reference for osteosarcoma treatment.

\section{Declarations}

\section{Consent for publication}

Not applicable

\section{Competing interests}

All authors report no conflicts of interest in this work.

\section{Funding}

This work was supported by the Youth Program of National Science Foundation of Zhejiang Province (Yefeng Xu, No. LQ18H290001), Traditional Chinese Medicine Science Research and Technology Program of Zhejiang Province (Yefeng Xu, No.2018ZB107), Hangzhou Health Science and Technology program 
project (Yefeng Xu, No. 2018A30), Hangzhou Agricultural and Social Development Research Project (Qingying Yan, No.20201203B215)

\section{Authors' contributions}

Network pharmacological analysis was prepared by Q.Y, J.Y and J.Z. QY, Y.Y and Y.W wrote the manuscript. M.C revised the submitted manuscript for important intellectual content. $X Y$ and $Y X$ designed the study and MS directed the study.

\section{References}

1. Ritter J, Bielack SS. Osteosarcoma. Ann Oncol. 2010; Suppl 7: vii320-5.

2. Meltzer PS, Helman LJ. New Horizons in the Treatment of Osteosarcoma. N Engl J Med. 2021; 385(22): 2066-2076.

3. Isakoff MS, Bielack SS, Meltzer P, Gorlick R. Osteosarcoma: Current Treatment and a Collaborative Pathway to Success. J Clin Oncol. 2015; 33(27): 3029-3035.

4. Argenziano M, Tortora C, Pota E, Di Paola A, Di Martino M, Di Leva C, Di Pinto D, Rossi F. Osteosarcoma in Children: Not Only Chemotherapy. Pharmaceuticals (Basel). 2021; 14(9): 923.

5. Zhang X, Qiu H, Li C, Cai P, Qi F. The positive role of traditional Chinese medicine as an adjunctive therapy for cancer. Biosci Trends. 2021; 15(5): 283-298.

6. Jinlong Ru, Peng Li, Jinan Wang, Wei Zhou, Bohui Li, Chao Huang, Pidong Li, Zihu Guo, Weiyang Tao, Yinfeng Yang, Xue Xu, Yan Li, Yonghua Wang, Ling Yang. TCMSP: a database of systems pharmacology for drug discovery from herbal medicines. J Cheminformatics. 2014; 6(1):13.

7. Xia Wang, Yihang Shen, Shiwei Wang, Shiliang Li, Weilin Zhang, Xiaofeng Liu, Luhua Lai, Jianfeng Pei, Honglin Li. PharmMapper 2017 update: a web server for potential drug target identification with a comprehensive target pharmacophore database. Nucleic Acids Res. 2017; 45, W356-W360.

8. Whirl-Carrillo M, Huddart R, Gong L, Sangkuhl K, Thorn CF, Whaley R, Klein TE. An Evidence-Based Framework for Evaluating Pharmacogenomics Knowledge for Personalized Medicine. Clin Pharmacol Ther. 2021; 110(3): 563-572.

9. Piñero J, Bravo À, Queralt-Rosinach N, Gutiérrez-Sacristán A, Deu-Pons J, Centeno E, García-García J, Sanz F, Furlong LI. DisGeNET: a comprehensive platform integrating information on human diseaseassociated genes and variants. Nucleic Acids Res. 2017; 45(D1): D833-D839.

10. Maleki Dana P, Sadoughi F, Asemi, Yousefi B. Anti-cancer properties of quercetin in osteosarcoma. Cancer Cell Int. 2021; 21(1): 349.

11. Wang $Y$, Kong D, Wang X, Dong X, Tao Y, Gong H. Molecular mechanisms of luteolin induced growth inhibition and apoptosis of human osteosarcoma cells. Iran J Pharm Res. 2015; 14(2): 531-8.

12. Zhang $B, Y u X, X i a ~ H$. The flavonoid luteolin enhances doxorubicin-induced autophagy in human osteosarcoma U2OS cells. Int J Clin Exp Med. 2015; 8(9): 15190-15197. 
13. Jiang L, Zhao X, Xu J, Li C, Yu Y, Wang W, Zhu L. The Protective Effect of Dietary Phytosterols on Cancer Risk: A Systematic Meta-Analysis. J Oncol. 2019; 2019:7479518.

14. Chauhan S, Sharma A, Upadhyay NK, Singh G, Lal UR, Goyal R. In-vitro osteoblast proliferation and in-vivo anti-osteoporotic activity of Bombax ceiba with quantification of Lupeol, gallic acid and $\beta$ sitosterol by HPTLC and HPLC. BMC Complement Altern Med. 2018; 18(1): 233.

15. Huang WW, Chiu YJ, Fan MJ, Lu HF, Yeh HF, Li KH, Chen PY, Chung JG, Yang JS. Kaempferol induced apoptosis via endoplasmic reticulum stress and mitochondria-dependent pathway in human osteosarcoma U-2 OS cells. Mol Nutr Food Res. 2010; 54(11):1585-1595.

16. Chen HJ, Lin CM, Lee CY, Shih NC, Peng SF, Tsuzuki M, Amagaya S, Huang WW, Yang JS. Kaempferol suppresses cell metastasis via inhibition of the ERK-p38-JNK and AP-1 signaling pathways in U-2 OS human osteosarcoma cells. Oncol Rep. 2013; 30(2): 925-932.

17. Marei HE, Althani A, Afifi N, Hasan A, Caceci T, Pozzoli G, Morrione A, Giordano A, Cenciarelli C. p53 signaling in cancer progression and therapy. Cancer Cell Int. 2021;21(1):703.

18. Synoradzki KJ, Bartnik E, Czarnecka AM, Fiedorowicz M, Firlej W, Brodziak A, Stasinska A, Rutkowski P, Grieb P. TP53 in Biology and Treatment of Osteosarcoma. Cancers (Basel). 2021; 13(17): 4284.

19. Chen Z, Guo J, Zhang K, Guo Y. TP53 Mutations and Survival in Osteosarcoma Patients: A MetaAnalysis of Published Data. Dis Markers. 2016; 2016:4639575.

20. Aleshin A, Finn RS. SRC: a century of science brought to the clinic. Neoplasia. 2010; 12(8): 599-607.

21. Hu C, Deng Z, Zhang Y, Yan L, Cai L, Lei J, Xie Y. The prognostic significance of Src and p-Src expression in patients with osteosarcoma. Med Sci Monit. 2015; 21: 638-645.

22. Baird K, Glod J, Steinberg SM, Reinke D, Pressey JG, Mascarenhas L Federman N, Marina N, Chawla S, Lagmay JP, Goldberg J, Milhem M, Loeb DM, Butrynski JE, Turpin B, Staddon A, Spunt SL, Jones RL, Tobogganer AND Schuetze SM, Okuno SH, Helman L. Results of a Randomized, Double-Blinded, Placebo-Controlled, Phase 2.5 Study of Saracatinib (AZD0530), in Patients with Recurrent Osteosarcoma Localized to the Lung. Sarcoma. 2020; 2020: 7935475.

23. Khalid AB, Krum SA. Estrogen receptors alpha and beta in bone. Bone. 2016; 87: 130-135.

24. Sonaglio V, de Carvalho AC, Toledo SR, Salinas-Souza C, Carvalho AL, Petrilli AS, et al. Aberrant DNA methylation of ESR1 and p14ARF genes could be useful as prognostic indicators in osteosarcoma. Onco Targets Ther. 2013; 6: 713-723.

25. Zhang J, Yu XH, Yan YG, Wang C, Wang WJ. PI3K/Akt signaling in osteosarcoma. Clin Bird Acta. 2015; 444: 182-192.

26. Xue Y, Zhang G, Zhou S, Wang S, Lv H, Zhou L, Shang P. Iron Chelator Induces Apoptosis in Osteosarcoma Cells by Disrupting Intracellular Iron Homeostasis and Activating the MAPK Pathway. Int J Mol Sci. 2021; 22(13): 7168.

27. Zhang L, Yang C, Huang Y, Huang H, Yuan X, Zhang P, Ye C, Wei M, Wang Y, Luo X, Luo J. Cardamonin inhibits the growth of human osteosarcoma cells through activating P38 and JNK signaling pathway. Biomed Pharmacother. 2021; 134:111155. 
28. Honorati MC, Cattini L Porters A. Possible prognostic role of IL-17R in osteosarcoma. J Cancer Res Clin Oncol. 2007; 133(12): 1017-1021.

\section{Tables}


Table 1

The information of 38 active ingredients.

\begin{tabular}{|c|c|c|c|c|}
\hline Mol ID & Molecule Name & OB\% & DL & HL \\
\hline MOL001663 & $\begin{array}{l}\text { (4aS,6aR,6aS,6bR,8aR,10R,12aR,14bS)-10- } \\
\text { hydroxy-2,2,6a,6b,9,9,12a-heptamethyl- } \\
\text { 1,3,4,5,6,6a,7,8,8a,10,11,12,13,14b- } \\
\text { tetradecahydropicene-4a-carboxylic acid }\end{array}$ & 32.0280133 & 0.75713 & 4.337611 \\
\hline MOL001659 & Poriferasterol & 43.8298516 & 0.75596 & 5.341727 \\
\hline MOL001670 & 2-methoxy-3-methyl-9,10-anthraquinone & 37.8277056 & 0.20517 & 28.820662 \\
\hline MOL001646 & 2,3-dimethoxy-6-methyanthraquinone & 34.8586047 & 0.26255 & 28.975305 \\
\hline MOL000211 & Mairin & 55.3770734 & 0.7761 & 8.873708 \\
\hline MOL000239 & Jaranol & 50.8288168 & 0.29148 & 15.50148 \\
\hline MOL000296 & hederagenin & 36.9139058 & 0.75072 & 5.347511 \\
\hline MOL000033 & $\begin{array}{l}\text { (3S,8S,9S,10R,13R,14S,17R)-10,13-dimethyl- } \\
\text { 17-[(2R,5S)-5-propan-2-yloctan-2- } \\
\text { yl]-2,3,4,7,8,9,11,12,14,15,16,17- } \\
\text { dodecahydro-1H- } \\
\text { cyclopenta[a]XXXhenanthrene-3-ol }\end{array}$ & 36.2284706 & 0.78288 & 5.217923 \\
\hline MOL000354 & isorhamnetin & 49.6043771 & 0.306 & 14.339263 \\
\hline MOL000371 & 3,9-di-O-methylnissolin & 53.7415267 & 0.47573 & 8.996019 \\
\hline MOL000379 & $\begin{array}{l}\text { 9,10-dimethoxypterocarpan-3-O-ß-D- } \\
\text { glucoside }\end{array}$ & 36.736688 & 0.9243 & 13.063156 \\
\hline MOL000380 & $\begin{array}{l}\text { (6aR,11aR)-9,10-dimethoxy-6a,11a-dihydro- } \\
\text { 6H-benzofurano[3,2-c]chromen-3-ol }\end{array}$ & 64.2554545 & 0.42486 & 8.493699 \\
\hline MOL000387 & Bifendate & 31.0978239 & 0.66553 & 17.961941 \\
\hline MOL000392 & formononetin & 69.6738806 & 0.21202 & 17.036852 \\
\hline MOL000398 & isoflavanone & 109.986656 & 0.29572 & 15.507494 \\
\hline MOL000417 & Calycosin & 47.7518278 & 0.24278 & 17.096724 \\
\hline MOL000422 & kaempferol & 41.8822495 & 0.24066 & 14.743371 \\
\hline MOL000433 & FA & 68.9604362 & 0.7057 & 24.811237 \\
\hline MOL000442 & 1,7-Dihydroxy-3,9-dimethoxy pterocarpene & 39.0454111 & 0.47943 & 7.946297 \\
\hline MOL000098 & quercetin & 46.4333481 & 0.27525 & 14.400548 \\
\hline MOL004355 & Spinasterol & 42.9793655 & 0.75534 & 5.321195 \\
\hline MOL001689 & acacetin & 34.9735727 & 0.24082 & 17.248472 \\
\hline
\end{tabular}




\begin{tabular}{|lllll|}
\hline Mol ID & Molecule Name & OB\% & DL & HL \\
\hline MOL000006 & luteolin & 36.1626293 & 0.24552 & 15.944492 \\
\hline MOL004580 & cis-Dihydroquercetin & 66.4369979 & 0.27344 & 14.513484 \\
\hline MOL005996 & $\begin{array}{l}\text { 2-O-methyl-3-O-3-D-glucopyranosyl } \\
\text { platycogenate A }\end{array}$ & 45.1502334 & 0.25226 & 6.025842 \\
\hline MOL006026 & $\begin{array}{l}\text { dimethyl 2-O-methyl-3-O-a-D-glucopyranosyl } \\
\text { platycogenate A }\end{array}$ & 39.2075766 & 0.25368 & 5.037639 \\
\hline MOL006070 & robinin & 39.8437311 & 0.70731 & 16.672864 \\
\hline MOL001323 & Sitosterol alpha1 & 43.2812704 & 0.78354 & 5.640765 \\
\hline MOL001494 & Mandenol & 41.9962005 & 0.19321 & 5.385969 \\
\hline MOL002882 & $\begin{array}{l}\text { [(2R)-2,3-dihydroxypropyl] (Z)-octadec-9- } \\
\text { enoate }\end{array}$ & 34.1310776 & 0.29824 & 5.189662 \\
\hline MOL000359 & sitosterol & 36.9139058 & 0.7512 & 5.371091 \\
\hline MOL000449 & Stigmasterol & 43.8298516 & 0.75665 & 5.574595 \\
\hline MOL008121 & 2-Monoolein & 34.2349738 & 0.29162 & 4.41187 \\
\hline MOL000953 & CLR & 37.8738975 & 0.67677 & 4.518834 \\
\hline MOL000358 & beta-sitosterol & 36.9139058 & 0.75123 & 5.355491 \\
\hline MOL004440 & Peimisine & 57.4023933 & 0.8055 & 14.39177 \\
\hline MOL004444 & Ziebeimine & $\begin{array}{l}\text { n-Methoxyl-2-acetyl-3-methyl-1,4- } \\
\text { glucopyranoside }\end{array}$ & 0.70486 & 7.809565 \\
\hline MOL004446 & & 0.57257 & 31.005736 \\
\hline
\end{tabular}


Table 2

The final values of $B C, C C, E C, D C$ and $L A C$ in core network.

\begin{tabular}{|llllll|}
\hline name & Betweenness & Closeness & Degree & Eigenvector & LAC \\
\hline TP53 & 5.521429 & 1 & 10 & 0.371665 & 6.4 \\
\hline SRC & 4.771429 & 0.909091 & 9 & 0.336607 & 5.555556 \\
\hline ESR1 & 4.169048 & 0.909091 & 9 & 0.340247 & 5.777778 \\
\hline HSP90AA1 & 2.935714 & 0.833333 & 8 & 0.308101 & 5.25 \\
\hline MYC & 2.438095 & 0.833333 & 8 & 0.309834 & 5.5 \\
\hline JUN & 2.002381 & 0.833333 & 8 & 0.313054 & 5.25 \\
\hline MAPK1 & 1.535714 & 0.769231 & 7 & 0.279498 & 5.142857 \\
\hline CDKN1A & 1.269048 & 0.769231 & 7 & 0.277749 & 4.857143 \\
\hline AKT1 & 0.821429 & 0.769231 & 7 & 0.288141 & 4.857143 \\
\hline CCND1 & 0.535714 & 0.714286 & 6 & 0.248775 & 4.333333 \\
\hline EGFR & 0 & 0.666667 & 5 & 0.20902 & 4 \\
\hline
\end{tabular}

Table 3 is available in the Supplemental Files section.

Figures 


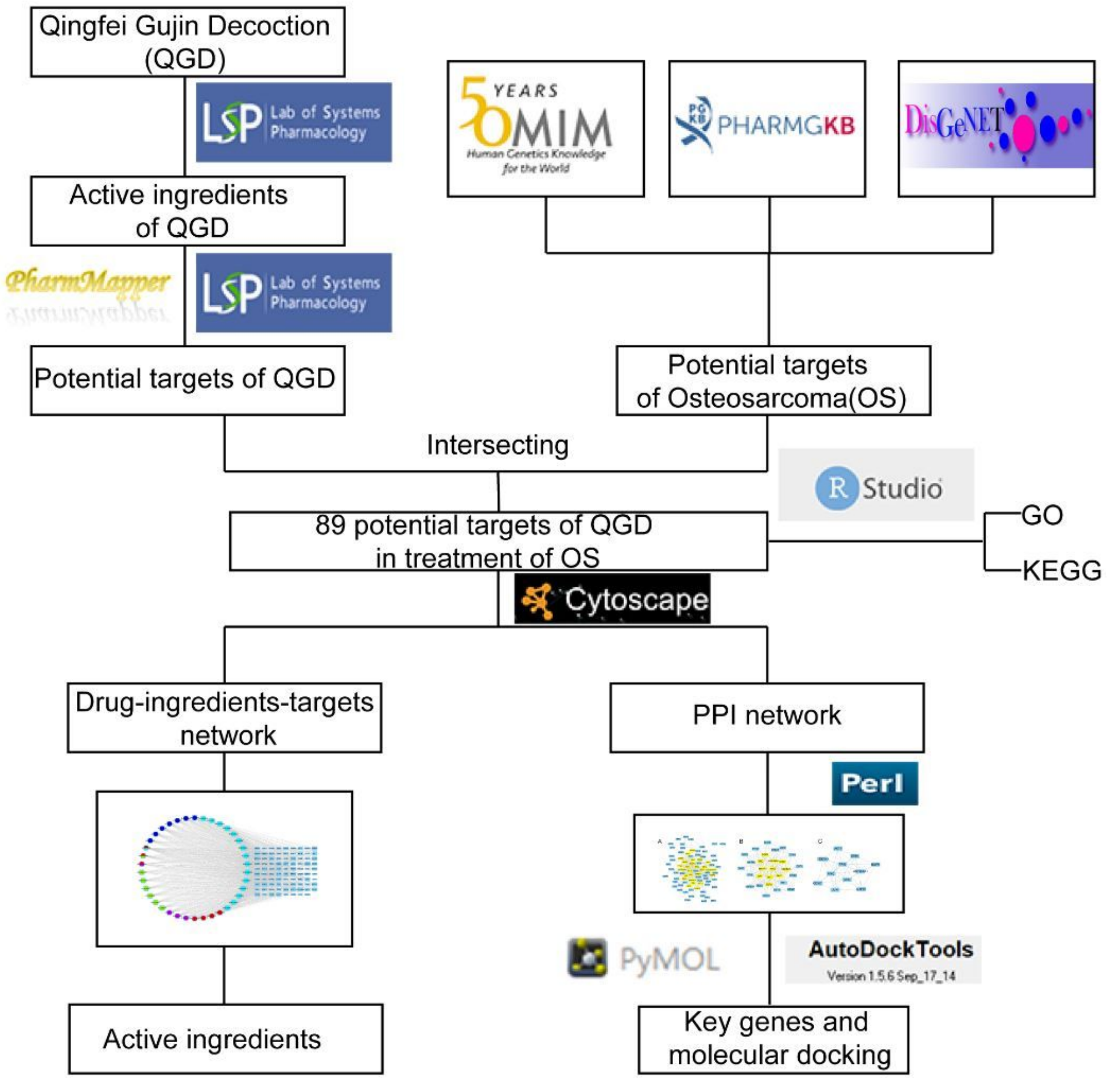

Figure 1

The flowchart of network pharmacology and molecular docking. 

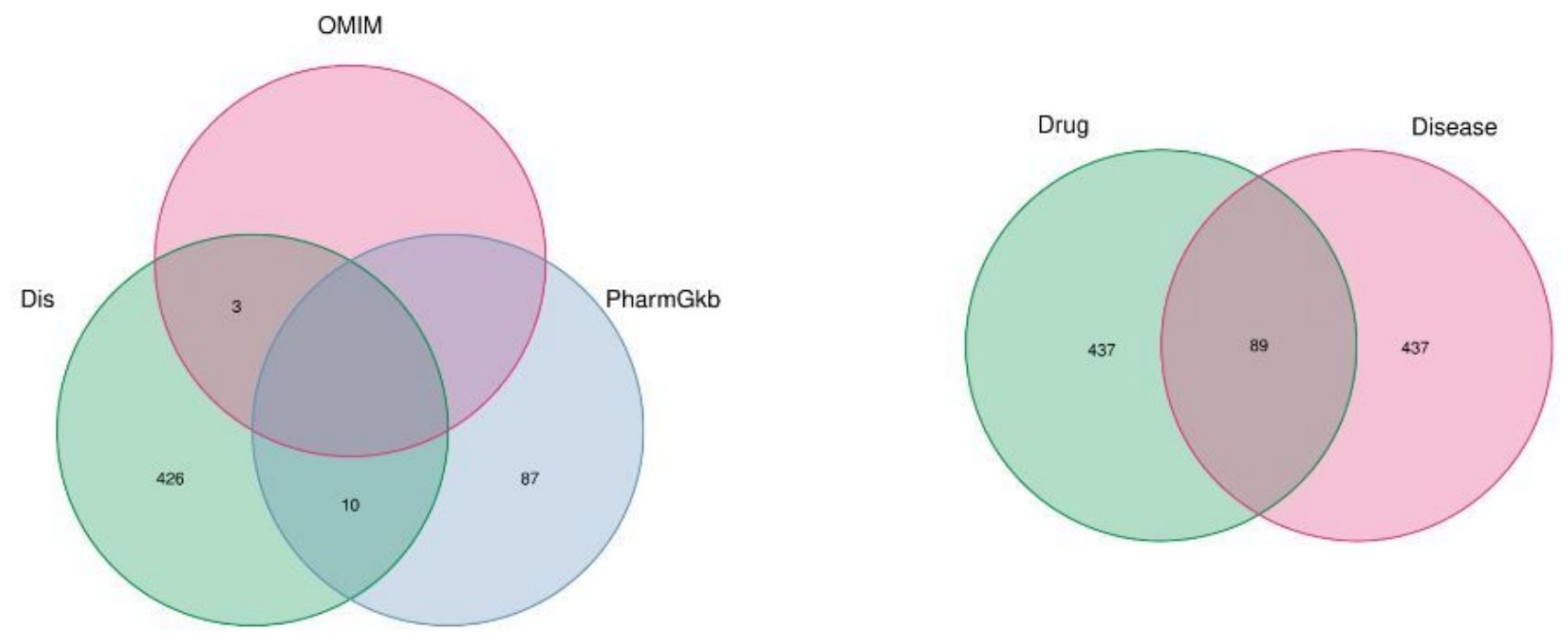

C

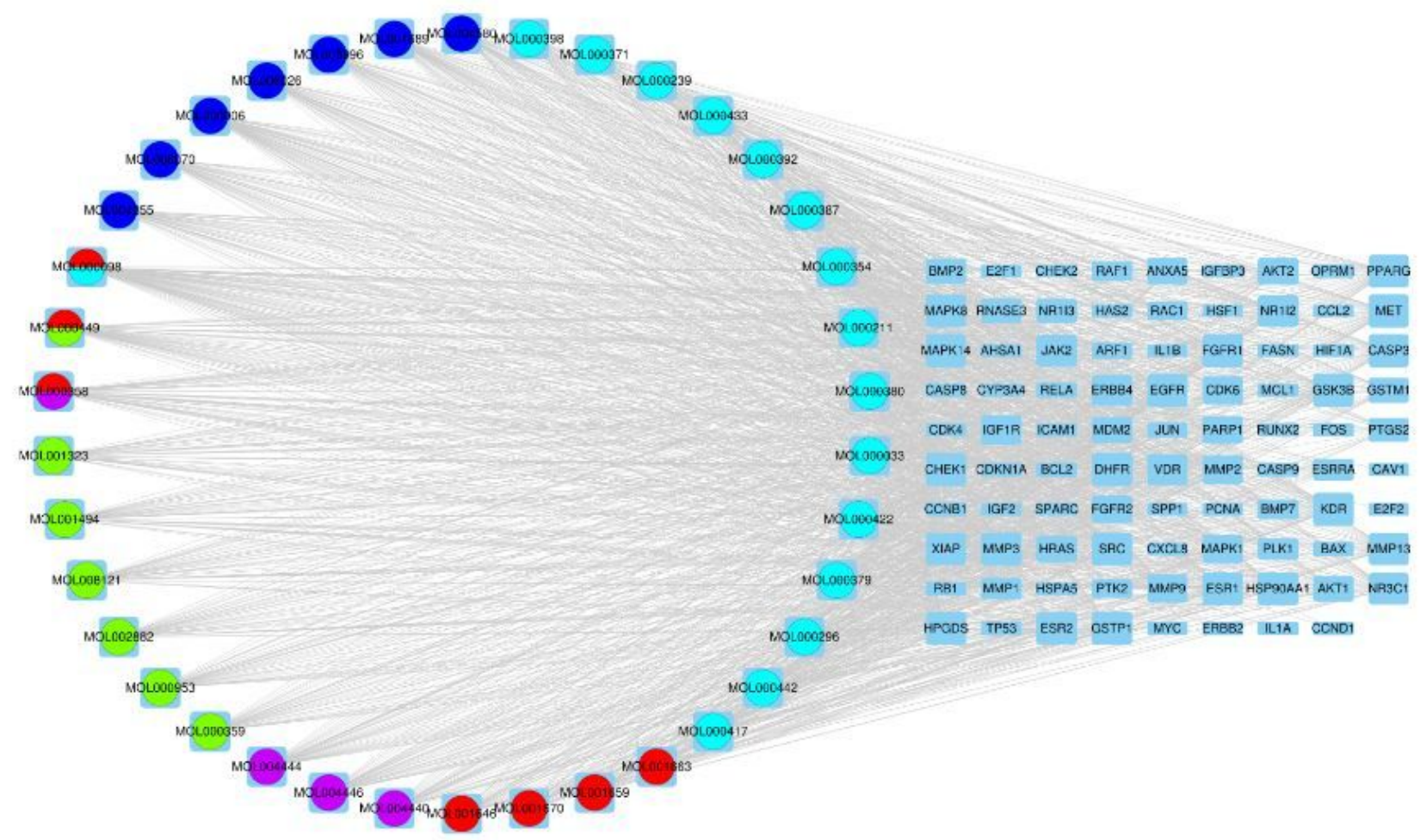

Figure 2

Potential targets and Drug-ingredients-targets network. (A) All the potential targets of OS. (B)Intersecting genes between drug and disease. (C) Drug-ingredients-targets network of QGD acting on OS. 

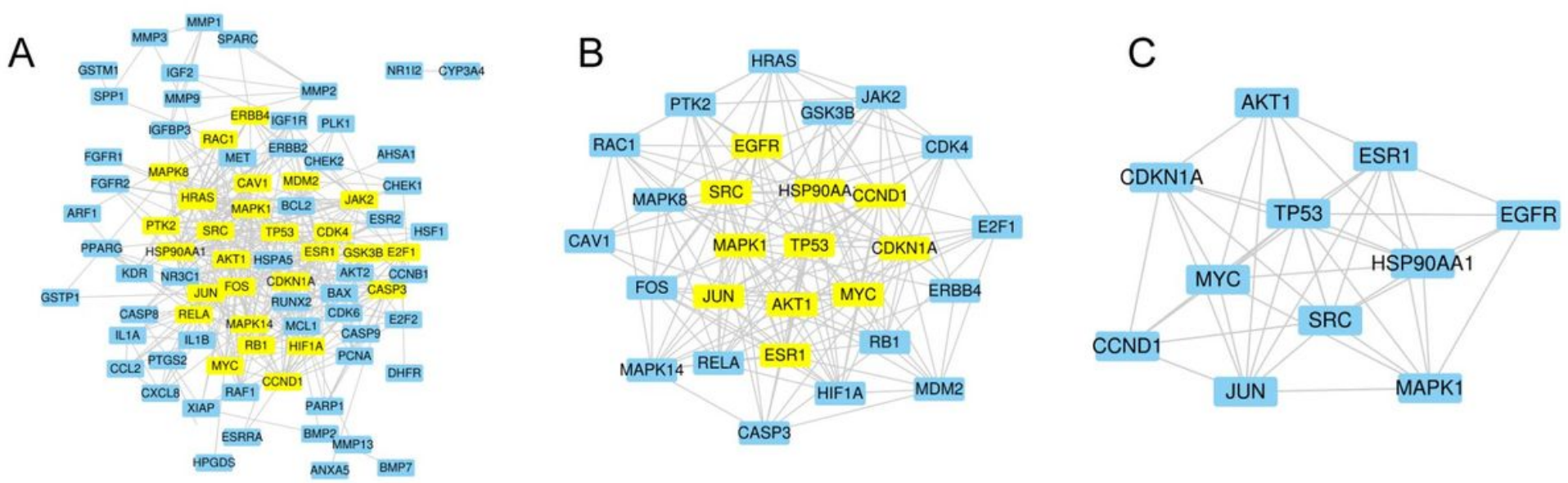

\section{Figure 3}

The screening of core network. (A)The original PPI network. (B) Sub-network. (C) The core network.

A

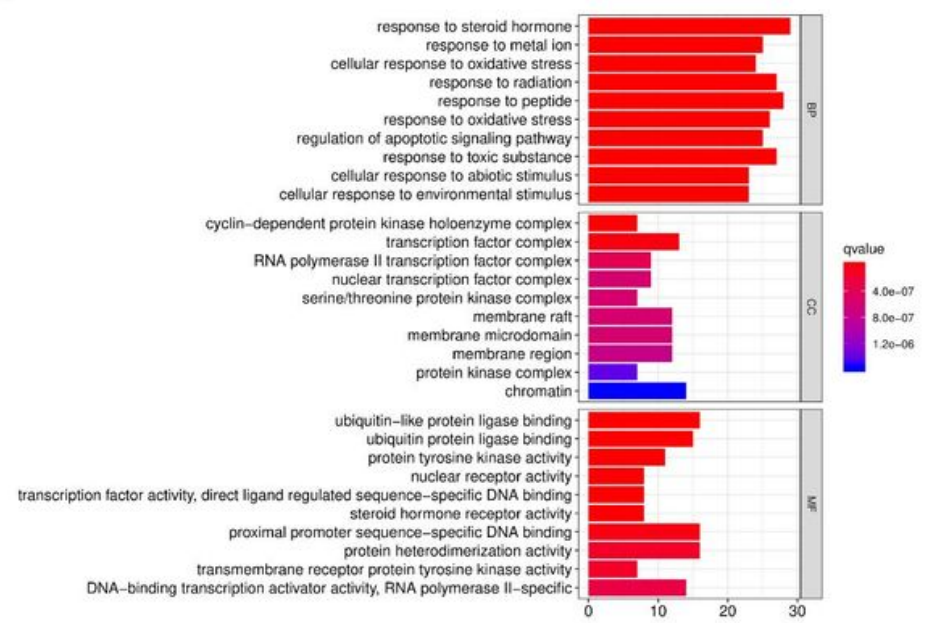

B

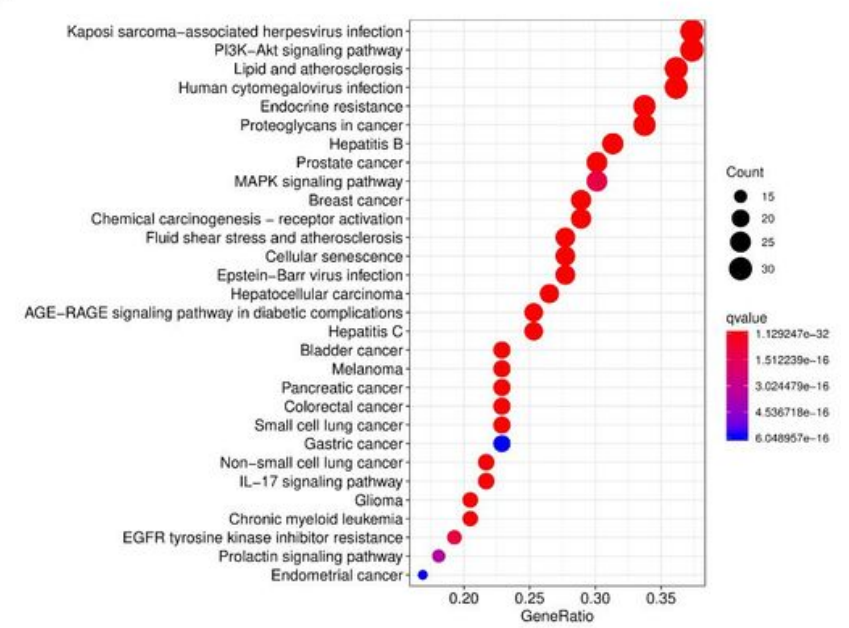

Figure 4

Enrichment analysis on 89 potential targets. (A) The barplot of GO enrichment. (B) The KEGG bubble. 
A

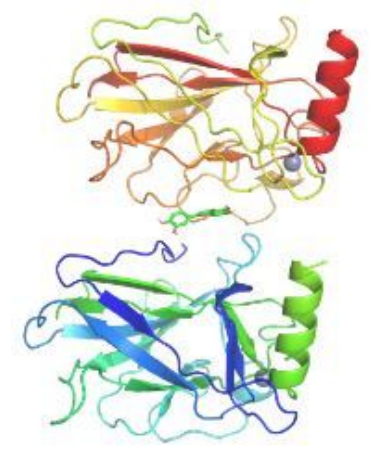

B
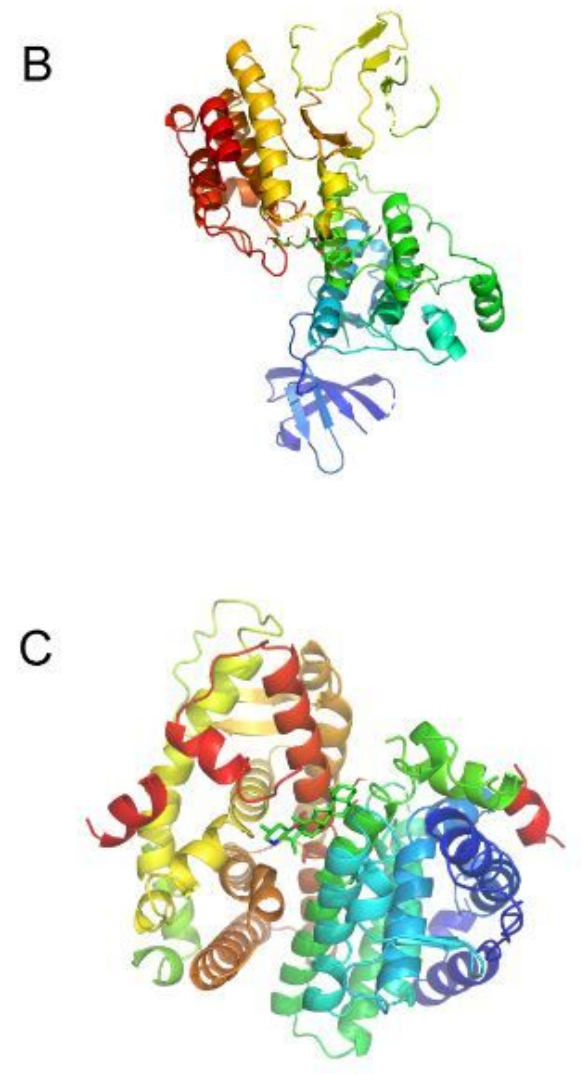
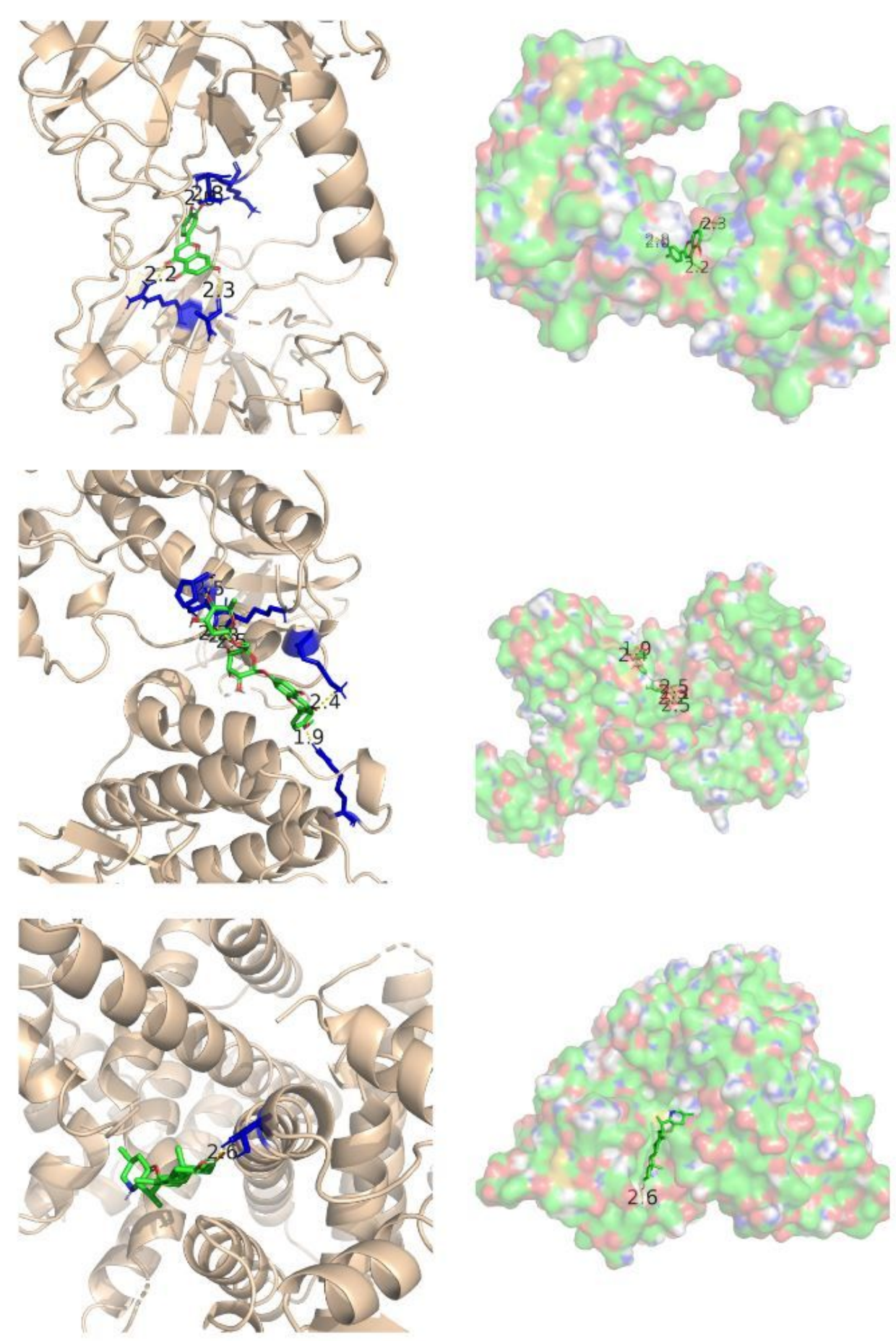

Figure 5

Molecular docking results. (A) Structure with an initial ligand of luteolin (MOL000006) with TP53(PDB ID: 5o1h). (B). Structure with an initial ligand of robinin (MOL006070) with SRC (PDB ID: 1yoj). (C) Structure with an initial ligand of Peimisine (MOL004440) with ESR1(PDB ID: 4tuz).

\section{Supplementary Files}


This is a list of supplementary files associated with this preprint. Click to download.

- Table3.jpg

- Supplementtable1Activeingredientsandcorrespondingtargets.docx

- Supplementtable2Themoleculardockingresultsbetweenthekeytargetsandtheactiveingredients.docx 